\title{
Mortalidad embrionaria tardía en ganado lechero altoandino
}

\author{
Study of late embryo mortality in dairy cattle from highland
}

\author{
Carlos Arana ${ }^{1,5}$, Olger Ramos ${ }^{2}$, Nidia Llapapasca ${ }^{3}$, Alfredo Delgado ${ }^{4}$
}

\section{Resumen}

La crianza de ganado lechero en las regiones altoandinas se encuentra limitada por el medio ambiente hipóxico, que puede afectar la supervivencia embrionaria. El presente estudio tuvo por objetivo determinar la mortalidad embrionaria (ME) tardía (28, 35 y 42 días pos-servicio) y su asociación con el número de partos $(0,1,2,3,4, \geq 5$ partos) y la producción láctea (60 y 90 días) en vacas Brown Swiss, Holstein y cruzadas, bajo un sistema de crianza semi-intensiva al pastoreo en el Valle del Mantaro a $3300 \mathrm{msnm}$. La ME tardía general fue de 7\% (14/203). Se encontró una asociación entre el número de partos y la presentación de $\mathrm{ME}(\mathrm{r}=0.8)$, así como entre la producción láctea y la $\mathrm{ME}(\mathrm{r}=0.9)$. La ME tardía en las vacas del valle del Mantaro puede considerarse como baja, y existiría una relación muy estrecha con los factores raza y número de partos.

Palabras clave: altitud, ganado lechero, mortalidad embrionaria, Holstein, Brown Swiss, cruzadas

${ }^{1,5}$ Estación Experimental del Centro de Investigación IVITA - El Mantaro, Jauja, Facultad de Medicina Veterinaria, Universidad Nacional Mayor de San Marcos, Huancayo, Perú

2 Estación Experimental del Centro de Investigación IVITA - Huaral, Facultad de Medicina Veterinaria, Universidad Nacional Mayor de San Marcos, Lima, Perú

3 Estación Experimental del Centro de Investigación IVITA - Pucallpa, Facultad de Medicina Veterinaria, Universidad Nacional Mayor de San Marcos, Lima, Perú

${ }^{4}$ Clínica de Animales Mayores, Facultad de Medicina Veterinaria, Universidad Nacional Mayor de San Marcos, Lima, Perú

5 E-mail: caranadel@unmsm.edu.pe; https://orcid.org/0000-0002-0668-3629

Recibido: 21 de enero de 2021

Aceptado para publicación: 15 de abril de 2021

Publicado: 23 de junio de 2021

CLos autores. Este artículo es publicado por la Rev Inv Vet Perú de la Facultad de Medicina Veterinaria, Universidad Nacional Mayor de San Marcos. Este es un articulo de acceso abierto, distribuido bajo los términos de la licencia Creative Commons Atribución 4.0 Internacional (CC BY 4.0) [https:// creativecommons.org/licenses/by/4.0/deed.es] que permite el uso, distribución y reproducción en cualquier medio, siempre que la obra original sea debidamente citada de su fuente original 
Dairy cattle breeding in the high Andean regions is limited due to the hypoxic environment, which may affect embryonic survival. The present study aimed to determine late embryonic mortality (EM) (28-, 35- and 42-days post-service) and its association with the number of calvings $(0,1,2,3,4, \geq 5)$ and milk yield (at 60 and 90 days) in Brown Swiss, Holstein and crossbred cows, under a semi-intensive rearing system at grazing in the Mantaro Valley (3300 meters above sea level). The overall late EM was 7\% (14/203). An association was found between the number of calvings and the presentation of EM $(\mathrm{r}=0.8)$, as well as between milk yield and $\operatorname{EM}(\mathrm{r}=0.9)$. The late ME in the cows of the Mantaro valley can be considered as low, and there would be a very close relationship with the factors of breed and parity.

Key words: highlands, dairy cows, embryo mortality, Holstein, Brown, crossbreed

\section{INTRODUCCIÓN}

La leche es un elemento básico para la seguridad alimentaria, siendo de $120 \mathrm{~L} /$ año el consumo per cápita recomendado por la FAO, pero en Perú el consumo no se supera los 87 L/persona/año. (MIDAGRI, 2020). La producción lechera en el país ha ido incrementándose con el transcurso de los años; sin embargo, aún se tienen problemas reproductivos como los abortos durante el primer tercio de la gestación, lo que implica pérdidas económicas para el productor y determina la posible salida de la vaca de la explotación (Sartori, 2004).

Uno de los problemas reproductivos más frecuentes es la mortalidad embrionaria (ME) que ocurre durante los primeros 45 días de gestación (Radostits et al., 2002; Santos et al., 2004). La mortalidad embrionaria es un serio inconveniente para el rendimiento reproductivo de las vacas; no obstante, estas vacas también pueden presentar alteraciones en la función ovárica que conducen a un retraso en la presentación del celo y en las posibilidades de volver a ser inseminadas (Remnant et al., 2015). De otra parte, los problemas de fertilidad y muerte embrionaria pueden deberse, en parte, a una respuesta a los tratamientos hormonales de inducción y sincronización del celo (Giordano et al., 2015).

La ME es uno de los problemas más difíciles de diagnosticar y corregir en reproducción bovina y puede presentarse debido a factores como el mal manejo, estrés, problemas de salud animal, entre otros (Diskin y Morris, 2008). La ME afecta el intervalo entre partos y la tasa de parición, así como una ralentización del progreso genético y pérdidas económicas en la producción lechera y de carne (Sartori, 2004). Se reportan pérdidas embrionarias durante los primeros 14 días del $30 \%$ de las concepciones sin que sean clínicamente detectadas (Dunne et al., 2000), siendo la mayoría (80\%) antes del octavo día, y de $5-10 \%$ entre los 14 y 19 días de la concepción (BonDurant, 2007; Diskin y Morris, 2008).

La muerte de embriones antes del reconocimiento materno de la gestación es considerada como ME temprana, la que ocurre entre el reconocimiento materno de la gestación y el momento que se ha completado la organogénesis se denomina ME tardía, y la pérdida de la gestación posterior se llama muerte fetal (Lonergan et al., 2016). Estudios que evaluaron la ME en bovinos con auxilio de la ecografía transrectal reportan 
resultados muy distintos, que en general se encuentran asociados a la raza, edad y lactación, entre otros (Vasconcelos et al., 1997).

En el Perú, en la zona de la costa se realizó un trabajo de $\mathrm{ME}$ determinándose que la mayor mortalidad ocurría en el día 28 del servicio (66.7\%) (Hernández, 2016). Sin embargo, estos estudios no han sido realizados en explotaciones altoandinas, de ahí que el objetivo del presente estudio fue determinar mediante exámenes ecográficos la tasa de ME tardía en animales diagnosticados como gestantes a partir de los 28 días posinseminación en vacas lecheras de altura, y su posible relación con la producción lechera y el número de partos.

\section{Materiales y Métodos}

El trabajo se realizó en establos de las provincias de Concepción y Jauja, situadas en el valle altoandino del Mantaro, sierra Central del Perú. La zona se encuentra a una altitud de $3300 \mathrm{msnm}$. Los establos fueron seleccionados por conveniencia, con similares características de crianza, manejo y empleo de inseminación artificial (IA).

Los animales fueron seleccionados con base a un historial reproductivo promedio para la zona y cuyo último parto fue sin alteraciones clínicas, y se les recabó la información de su producción lechera desde el parto hasta el día 42 pos-IA. Se trabajó con 203 vacas, siendo 103 Brown Swiss. 54 Holstein y 46 cruzadas. Todos los animales se encontraban bajo un sistema de crianza semi-intensiva, con instalaciones adecuadas de una crianza de la sierra peruana (Valle del Mantaro) y con alimentación a base de pastos cultivados, forraje y ensilado. En todos los casos se empleó la IA como método reproductivo. Los animales fueron clasificados según el número de partos $(0,1,2,3,4$, e»5).
La IA se realizó a la presentación del primer celo, superado el periodo de espera voluntario de 60 días posparto. El diagnóstico de gestación para detectar la posible presencia del embrión se realizó a los 28, 35 y 42 días del servicio mediante ecografía por el mismo profesional. Se utilizó un ecógrafo portátil Handscan V8 (Sunway Medical, China) con transductor rectal lineal a una frecuencia de $7.5 \mathrm{MHz}$.

Los datos de ME agrupados por número de partos y grupo racial son presentados como frecuencias. La asociación entre el número de partos y la presentación de $\mathrm{ME}$ se realizó mediante el análisis de correlación de Pearson ( $r$ ) al nivel de $p<0.05$. La producción láctea (promedio $\pm \mathrm{DE}$ ) a los 60 y 90 días de la lactación entre animales con y sin ME fueron comparados con la prueba de Tstudent al nivel de $\mathrm{p}<0.05$ y la asociación entre estos parámetros se determinó igualmente mediante la correlación de Pearson. Todos los análisis se hicieron con el software GraphPad Prism 6.0.

\section{Resultados}

La frecuencia de mortalidad embrionaria según el número de parto y el grupo racial de las vacas se presenta en la Figura 1 . Se encontró una asociación fuerte $(\mathrm{r}=0.8)$ entre el número de partos y la presentación de $\mathrm{ME}$ en el ganado lechero altoandino (Figura 1D). De manera similar, los ocho y seis casos de mortalidad embrionaria observados a los 35 y 42 días del servicio, con relación a las gestaciones confirmadas a los 28 días del servicio indicaron una correlación significativa $(\mathrm{r}=0.9)$ con una mayor producción de leche a los 60 y 90 días de lactación en animales que presentaron ME (Figura 2D). 

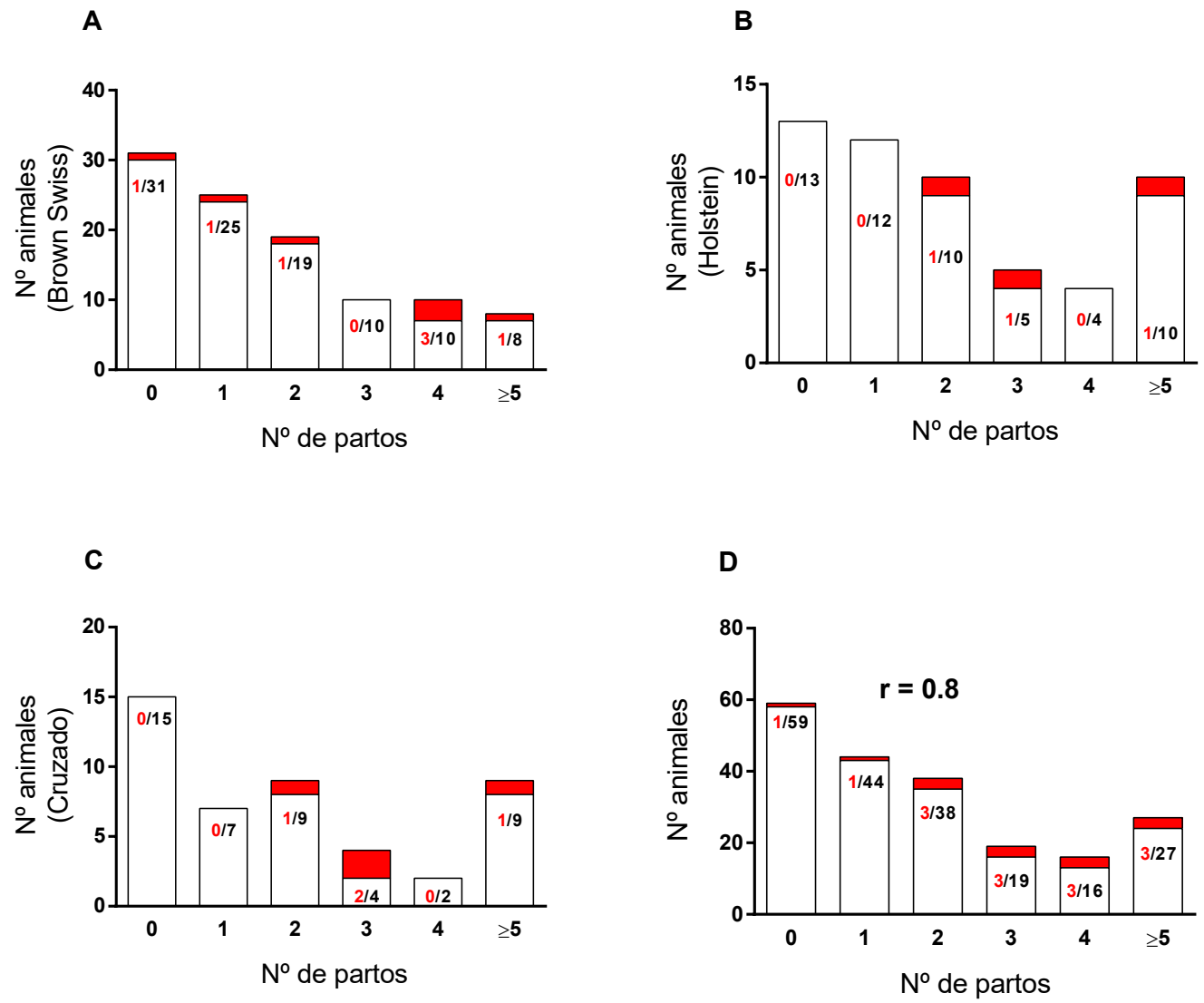

Figura 1. Ocurrencia de mortalidad embrionaria - ME (barras y números en rojo) en vacas agrupadas según el número de partos en vacas Brown Swiss (A), Holstein (B), cruzadas (C) y en el total de animales (D). Las correlaciones indican una asociación fuerte entre el número de partos y la $\mathrm{ME}(\mathrm{p}<0.05)$

\section{Discusión}

La crianza altoandina de ganado lechero está muy extendida a pesar de observarse diversos efectos negativos; así, el bovino Holstein es más susceptible al mal de altura que el Brown Swiss (Ayón et al., 1989), así como en la respuesta productiva por el tipo de alimentación y manejo que puede derivar a alteraciones relacionadas a la hipoxia de altura (Cueva et al., 1989; Lira et al., 2007). Sin embargo, en la actualidad se ha podido reducir significativamente la morbilidad y mortalidad causada por la altitud, haciendo de la ganadería lechera altoandina una actividad sostenible y rentable (Aubron y Cochet, 2009).
Si bien la concepción puede llegar al $85 \%$ en la mayoría de los casos, si es que la IA se realiza correctamente, una proporción significativa de los embriones resultantes no llega a término (Diskin y Morris, 2008; Wiltbank et al., 2016; Vanroose et al., 2000). De otra parte, la crianza de ganado lechero en altura así como en nivel de mar tienen los mismos problemas en el área reproductiva como la ME, aunque las presentaciones e incidencias podrían ser distintas. Los resultados del presente estudio indican una frecuencia de 7, 6 y 9\% en las vacas raza Brown Swiss, Holstein y cruzados, respectivamente, con un promedio global de $7 \%$ (14/203). Silke et al. (2002) cuantificaron la ME tardía a partir del día 28 de gestación en 1046 va- 
A

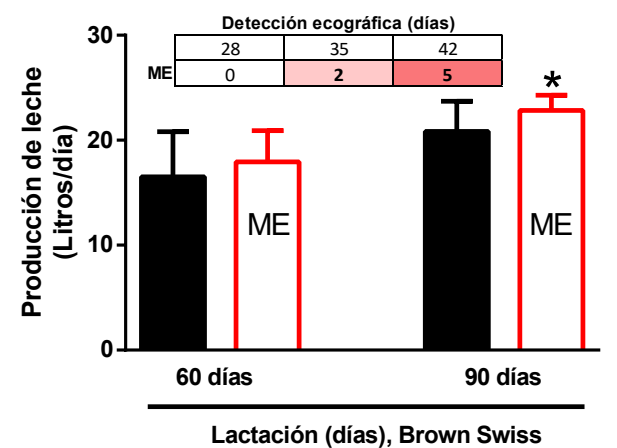

C

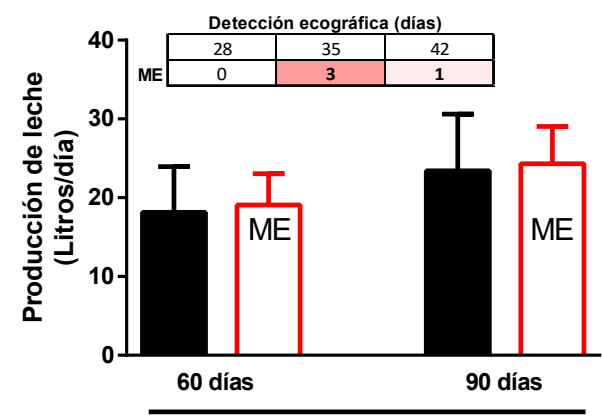

Lactación (días), Cruzado
B

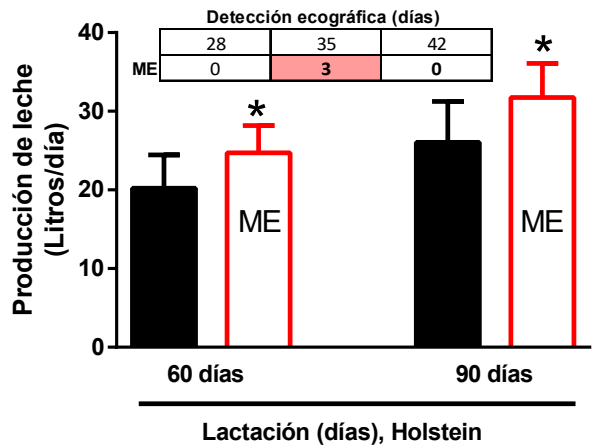

D

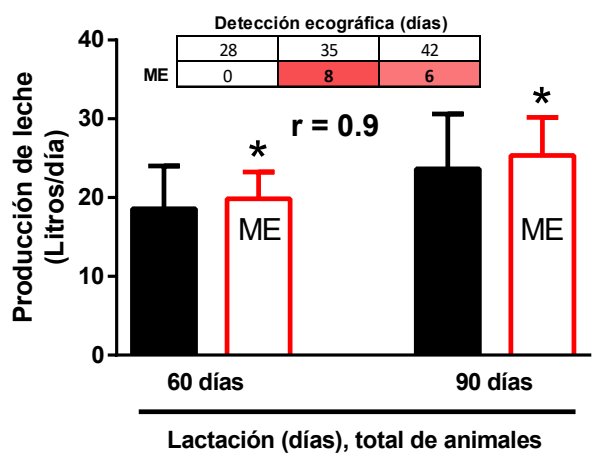

Figura 2. Ocurrencia de mortalidad embrionaria - ME (barras y números en rojo) en vacas agrupadas según la producción láctea en vacas Brown Swiss (A), Holstein (B), cruzadas (C) y en el total de animales (D). Los diagnósticos de gestación fueron realizados por ecografía a los 28,35 y 42 días pos-inseminación. * = Diferencia significativa entre animales con (barra roja) y $\sin$ (barra negra) $\mathrm{ME}(\mathrm{p}<0.05)$

cas lecheras y 162 novillas al pastoreo lecheras manejadas en sistemas de producción de leche basados en pasturas, encontrando tasas de pérdida embrionaria/fetal de 7.2 y $6.1 \%$, respectivamente, entre los 28 y 84 días del servicio, aunque no encontraron una relación con la producción de leche. En forma similar, Horan et al. (2004) registraron una tasa de ME tardía de $7.5 \%$ en vacas lecheras al pastoreo. Cabe resaltar que la ME tardía en sistemas al pastoreo es menor que la reportada en vacas de explotaciones intensivas (Vasconcelos et al., 1997; Santos et al., 2004).

Se encontró una asociación significativa entre la presentación de ME y los niveles de producción de leche para las vacas Holstein y Brown Swiss, pero no para las vacas cruzadas. Las vacas con ME produjeron más leche que aquellas que no perdieron el embrión ( $\mathrm{r}=0.9$; Figura 2$)$. 
La fuerte asociación $(r=0.8)$ entre el número de partos y la presentación de $\mathrm{ME}$ en ganado lechero altoandino tiene una estrecha relación con la edad de los animales. Según Vanroose et al. (2000), los animales más viejos tienen menor actividad folicular y menor calidad de ovocitos, lo que resulta en una disminución de la capacidad de desarrollo de los embriones. Además, la calidad del medio ambiente donde se desarrolla el embrión disminuye con la edad de la madre (Shorten et al., 2015).

Varios estudios han podido demostrar que la ME tardía podría estar relacionado a la actividad hormonal del tracto reproductivo de las vacas. En este sentido, Grimard et al. (2006) determinaron $25 \%$ de incidencia de ME tardía en 1285 vacas y que esta se encontraba asociada con la estacionalidad de la IA, el nivel de producción de leche y la condición corporal, pero no con el valor genético. Cunha et al. (2008) registraron $6.8 \%$ de $\mathrm{ME}$ en vacas con altas concentraciones de progesterona y $14.3 \%$ de $\mathrm{ME}$ en vacas con baja concentración de progesterona. Asimismo, otros estudios han registrado mayores tasas de pérdida de preñez en vacas lecheras anovulatorias en comparación con las cíclicas (Santos et al., 2004; Sterry et al., 2006; Stevenson et al., 2006), lo cual indicaría que concentraciones elevadas de progesterona en el ciclo previo a la IA tienen un efecto positivo en el mantenimiento de la gestación, incluso mucho después de la implantación.

Los cambios fisiológicos y metabólicos asociados con una alta producción de leche como la disminución (glucosa, insulina, IGFI) o el aumento (ácidos grasos no esterificados y cuerpos cetónicos) de metabolitos circulantes durante la distribución de nutrientes asociados con el balance energético negativo se han relacionado con una pobre eficiencia reproductiva (Walsh et al., 2011). También se ha propuesto que la alta producción lechera; es decir, los altos requerimientos energéticos, podría afectar la calidad de los ovocitos debido a un microambiente folicular deficiente, un entorno del tracto reproductivo subóptimo para el embrión y por una comunicación embrionaria inadecuada (Lonergan et al., 2016).

\section{Conclusiones}

- La mortalidad embrionaria (ME) tardía en las vacas del valle del Mantaro puede considerarse como baja.

- La ME tardía estuvo relacionada con el factor raza y con el número de partos

\section{Literatura Citada}

1. Aubron C, Cochet H. 2009. Producción lechera en los Andes peruanos: ¿Integración al mercado interno o marginación económica? Anuario Americanista Europeo. 6: 217-238.

2. Ayón M, Chauca D, Cueva S, Llerena L. 1989. Modalidad de crianza e incidencia de mal de altura en vacunos. En: XII Reunión Científica Anual de la Asociación de Producción Animal. Lima, Perú.

3. BonDurand R. 2007. Selected diseases and conditions associated with bovine conceptus loss in the first trimester. Theriogenology 68: 461-473. doi: 10.1016/j.theriogenology.2007.04.022

4. Cueva S, Ayón M, Chauca O. 1989. Producción láctea e incidencia de mal de altura en crías de vacas Holstein a 3320 m de altitud. En: XII Reunión Científica Anual de la Asociación Peruana de Producción Animal.

5. Cunha AP, Guenther JN, Maroney MJ, Giordano JO, Nascimento AB, Bas S, Ayres H, et al. 2008. Effects of high vs. low progesterone concentrations during Ovsynch on double ovulation rate and pregnancies per $\mathrm{AI}$ in high producing dairy cows. J Dairy Sci 91: 246. 
6. Diskin MG, Morris DG. 2008. Embryonic and early foetal losses in cattle and other ruminants. Reprod Domest Anim 43: 260-267. doi: 10.1111/j.14390531.2008.01171.x

7. Dunne LD, Diskin MG,Sreenan JM. 2000. Embryo and foetal loss in beef heifers beetwen day 14 of gestation and full term. Anim Repro Sci 58: 39-44. doi: 10.1016/s0378-4320(99)00088-3

8. Giordano J, Stangaferro M, Wijma R, Chandler W, Watters R. 2015. Reproductive performance of dairy cows managed with a program aimed at increasing insemination of cows in estrus based on increased physical activity and fertility of timed artificial inseminations. J Dairy Sci 98: 2488-2501. doi: 10.3168/ jds.2014-8961

9. Grimard B, Freret $S$, Chevallier A, Pinto A, Ponsart C, Humblot P. 2006. Genetic and environmental factors influencing first service conception rate and late embryonic/foetal mortality in low fertility dairy herds. Anim Reprod Sci 91: 31-44. doi: 10.1016/J.ANIREPROSCI. 2005.03.003

10. Hernández V. 2016. Determinación de mortalidad embrionaria por ultrasonografía en vacas Hosltein Friesian de la tercera a sétima semana en un establo de Cartavio Perú. Tesis de Médico Veterinario y Zootecnista. Trujillo, Perú: Univ. Privada Antenor Orrego. 38 p.

11. Horan B, Mee JF, Rath M, O'Connor P, Dillon P. 2004. The effect of strain of Holstein-Friesian cow and feeding system on reproductive performance in seasonal-calving milk production systems. Anim Sci 79: 453-467. doi: 10.1017/S1357729800090329

12. Lira B, Ayón M, Cueva S, Vásquez, M, Arana C, Ocampo N. 2007. Efecto del ketoprofeno sobre la presión arterial pulmonar en terneros Jersey sometidos a hipoxia de la altura. Rev Inv Vet Perú 18:94-98. doi: 10.15381/rivep.v18i2.1283
13. Lonergan P, Fair T, Forde N, Rizos D. 2016. Embryo development in dairy cattle. Theriogenology 86: 270-277. doi: 10.1016/j.theriogenology.2016.04.040

14. [MIDAGRI] Ministerio de Desarrollo Agrario y Riego. 2020. Minagri: 452 mil familias en el Perú se dedican a la producción de leche y sus derivados [Internet]. Disponible en: https:// www.gob.pe/institucion/midagri/noticias/ 168070-minagri-452-mil-familias-en-elperu-se-dedican-a-la-produccion-de-leche-y-sus-derivados

15. Radostits OM, Gay C, Blood D, Hinchliff K. 2002. Medicina veterinaria. $9^{\circ}$ ed. Vol I. Madrid, España: McGraw Hill Interamericana. 1206 p.

16. Remnant J, Green M, Huxley J, Hudson C. 2015. Variation in the interservice intervals of dairy cows in the United Kingdom. J Dairy Sci 98: 889897. doi: 10.3168/jds.2014-8366

17. Santos J, Thatcher W, Chebel R, Cerri R, Galvao K. 2004. The effect of embryonic death rates in cattle on the efficacy of estrus synchronization programs. Anim Reprod Sci 82: 513-535. doi: 10.1016/j.anireprosci.2004.04.015

18. Sartori R. 2004. Fertilização e morte embrionária em bovinos. Acta Sci Vet 32: $35-50$

19. Silke V, Diskin MG, Kenny DA, Boland MP, Dillon P, Mee JF, Sreenan JM. 2002. Extent, pattern and factors associated with late embryonic loss in dairy cows. Anim Reprod Sci 71: 1-12. doi: 10.1016/s0378-4320(02)00016-7

20. Shorten P, Morris CA, Cullen NG 2015. The effects of age, weight, and sire on pregnancy rate in cattle. J Anim Sci 93: 1535-1545. doi: $10.2527 /$ jas.2014-8490

21. Sterry RA, Welle ML, Fricke PM. 2006. Treatment with gonadotropinreleasing hormone after first timed artificial insemination improves fertility in noncycling lactating dairy cows. J Dairy Sci 89: 4237-4245. doi: 10.3168/ jds.S0022-0302(06)72469-9 
22. Stevenson JS, Pursley JR, Garverick HA, Fricke PM, Kesler DJ, Ottobre JS, Wiltbank MC. 2006. Treatment of cycling and noncycling lactating dairy cows with progesterone during Ovsynch. J Dairy Sci 89: 2567-2578. doi: 10.3168/ jds.S0022-0302(06)72333-5

23. Vanroose G, de Kruif A, Van Soom A. 2000. Embryonic mortality and embryopathogen interactions. Anim Reprod Sci 60: 131-143. doi: 10.1016/S03784320(00)00098-1

24. Vasconcelos JLM, Silcox RW, Lacerda JA, Pursley JR, Wiltbank MC. 1997. Pregnancy rate, pregnancy loss, and response to head stress after AI at 2 different times from ovulation in dairy cows. Biol Reprod 56: 140.
25. Vasconcelos JLM, Silcox RW, Lacerda JA, Pursley JR, Wiltbank MC. 1997. Pregnancy rate, pregnancy loss and response to heat stress after AI at 2 different times from ovulation in dairy cows. Biol Reprod 56: 140.

26. Walsh SW, Williams EJ, Evans AC. 2011. A review of the causes of poor fertility in high milk producing dairy cows. Anim Reprod Sci 123: 127-138. doi: 10.1016/j.anireprosci.2010.12.001

27. Wiltbank MC, Baez GM, Garcia-Guerra A, Toledo M Z, Monteiro PLJ, Melo LF, Sartori R. 2016. Pivotal periods for pregnancy loss during the first trimester of gestation in lactating dairy cows. Theriogenology 86: 239-253. doi: 10.1016/j.theriogenology.2016.04.037 